\title{
Letters
}

\section{Adenosine deaminase activity in rheumatoid pleural effusion}

SIR, We read with interest the article by Ocaña et al describing nine cases of rheumatoid pleural effusion, all with raised levels of adenosine deaminase activity in pleural fluid but none with specific changes in pleural biopsy. ${ }^{1}$ We have recently seen a rheumatoid patient who developed a pleural effusion with raised levels of adenosine deaminase and showed a typical rheumatoid nodule in the pleural biopsy specimen.

The patient, a 64 year old woman, had developed seropositive, nodular rheumatoid arthritis three years before admission. She had to stop gold salt injections because of a toxic skin reaction and she was receiving D-penicillamine at the time of admission to hospital, with good control of her arthritis.

Two months before admission she started a cough with mucoid sputum, mild left thoracic discomfort, and disturbed sensation. She also had mild constitutional symptoms. There was no flare up of her arthritis. A chest radiograph showed left pleural effusion. Physical examination showed an afebrile patient with signs of left pleural effusion, ulnar

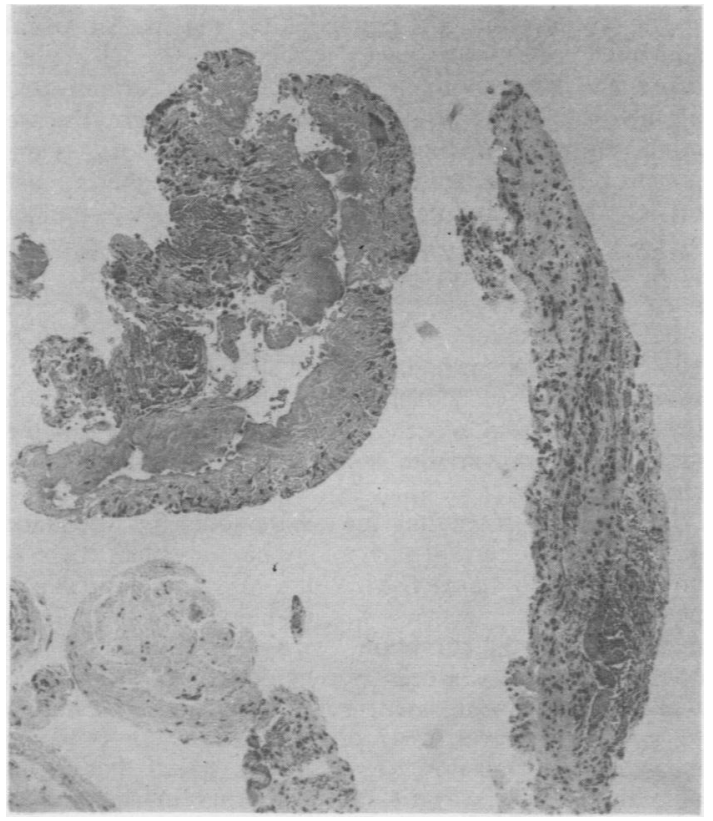

Fig. 1 Pleural biopsy specimen. Fragment of pleural tissue showing the presence of fibrinoid necrosis, surrounded by $a$ palisade of histiocytic cells and some multinucleated giant cells. Acid fast stains were negative.

(Haematoxylin and eosin.) deviation, synovial proliferation of her wrists, and mild arthritis in her knees. No subcutaneous nodules were found. The rest of the general examination was normal. Laboratory findings were: erythrocyte sedimentation rate $86 \mathrm{~mm} / \mathrm{h}$, haemoglobin $118 \mathrm{~g} / \mathrm{l}$, white blood cell count normal, platelets $540 \times 10^{9} / \mathrm{l}$. Blood chemistry and urine analysis were normal. Rheumatoid factor was 1.090 IU. Antinuclear factor was positive at a dilution of $1 / 6400$ with homogeneous pattern. Anti-dsDNA was negative.

Thoracentesis gave a yellowish fluid with $1 \cdot 15 \times 10^{9} \mathrm{cells} / \mathrm{l}$, $75 \%$ mononucleated and $25 \%$ polymorphonucleated. Glucose was $56 \mathrm{mmol} / \mathrm{l}$ and proteins $56 \mathrm{~g} / \mathrm{l}$. Adenosine deaminase activity increased to $89 \mathrm{U} / \mathrm{l}$. Pleural fluid cultures were negative and there were no malignant cells. Pleural biopsy with Abrams' needle showed the presence of a typical rheumatoid nodule (Fig. 1). Ziehl's stain and Löwenstein's culture of pleural tissue were negative.

An infectious or neoplastic aetiology was ruled out, treatment started with $8 \mathrm{mg}$ daily of methylprednisolone, and the patient's condition improved.

In the cases of rheumatoid pleural effusion reported by Ocaña $e a^{1}{ }^{12}$ a study of the pleural fluid did not show any difference from the usual findings in tuberculosis patients, including increased adenosine deaminase activity. Pleural biopsy was also non-specific in all the cases reported. Therefore the diagnosis of rheumatoid pleural effusion was made by exclusion.

In our patient, findings in the pleural fluid were consistent with those reported by Ocaña et al, ${ }^{12}$ but diagnosis of rheumatoid aetiology was reinforced by the presence of a typical rheumatoid nodule in the pleural biopsy specimen.

Departments of ${ }^{1}$ Rheumatology,

${ }^{2}$ Pneumology, and ${ }^{3}$ Pathology,

Hospital Ntra. Sra. de L'Esperança,

Barcelona, Spain

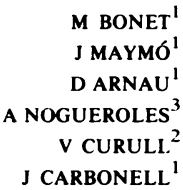

\section{References}

1 Ocaña I, Ribera E, Martinez-Vázquez J M, et al. Adenosine deaminase activity in rheumatoid pleural effusion. Ann Rheum Dis 1988; 47: 394-7.

2 Ocaña I, Martinez-Vázquez J M, Segura R M, Fernandez-DeSevilla T, Capdevila J A. Adenosine deaminase in pleural fluids. Test for diagnosis of tuberculosis pleural effusion. Chest 1983; 84: 51-3.

\section{Pulse methylprednisolone therapy in rheumatoid arthritis}

SIR, Over the last 12 months several publications about the use of pulse methylprednisolone therapy in the treatment of rheumatoid arthritis have appeared, reporting the 
efficacy and low incidence of side effects from this treatment. ${ }^{1-5}$ Two further papers appeared in the November issue of the Annals. ${ }^{67}$ The paper by Walters and Cawley showed the value of combining pulse methylprednisolone with sodium aurothiomalate treatment, ${ }^{6}$ results similar to our findings in a previous publication, of which the authors appeared not to be aware. ${ }^{3}$ The paper by Williams et al again showed the low incidence of adverse effects from this treatment and, more specifically, failed to find an increased incidence of avascular necrosis of the femoral head, which theoretically would be an expected adverse effect in view of the large doses of corticosteroid used in pulse methylprednisolone therapy. "lthough this retrospective cohort study needs to be confirmed by a prospective long term study with large patient numbers as well as matched controls, it confirms the favourable risk/benefit ratio of this treatment. It is therefore surprising that our papers on this subject in another journal ${ }^{3-5}$ led to an editorial comment ${ }^{8}$ and correspondence ${ }^{910}$ describing this treatment as 'ineffective' and 'hardly justifiable' in the treatment of rheumatoid arthritis. Perhaps this simply reflects a conservative attitude of North American clinicians, yet it is surprising that pulse therapy, with its favourable risk/benefit ratio, considerable efficacy in short term control of inflammation, and efficacy as oral treatment, ${ }^{11}$ should not be at least as attractive a therapeutic option as the alternatives-namely, non-steroidal anti-inflammatory drugs (low efficacy, high incidence of adverse effects) and disease modifying antirheumatic drugs (slow onset of action, high incidence of adverse effects, high drop out rate). None of the recent publications has advocated pulse therapy as an alternative to remittive agents, but rather as adjunctive treatment to achieve rapid control of inflammation while awaiting control with a remittive agent, or in the event of failed treatment with several disease modifying antirheumatic drugs.

Perhaps it is time for a Viewpoint article in the Annals on the place of pulse methylprednisolone therapy in the treatment of rheumatoid arthritis, lest a potentially useful treatment should be neglected as a result of therapeutic conservatism.

Arthritis Unit, MALCOLM D SMITH

Massachusetts General Hospital,

Boston,

Massachusetts,

USA

\section{References}

1 Needs C J, Smith M, Boutagy J, et al. Comparison of methylprednisolone ( $1 \mathrm{~g}$ IV) with prednisolone ( $1 \mathrm{~g}$ orally) in rheumatoid arthritis: a pharmacokinetic and clinical study. $J$ Rheumatol 1988; 15: 224-8.

2 Radia M, Furst D E. Comparison of three pulse methylprednisolone regimens in the treatment of rheumatoid arthritis. $J$ Rheumatol 1988; 15: 242-5.

3 Smith M D, Bertouch J V, Smith A M, et al. The clinical and immunological effects of pulse methylprednisolone therapy in rheumatoid arthritis. I. Clinical effects. J Rheumatol 1988; 15: 229-32.

4 Smith M D, Ahern M J, Brooks P M, et al. The clinical and immunological effects of pulse methylprednisolone therapy in rheumatoid arthritis. II. Effects on immune and inflammatory indices in peripheral blood. $J$ Rheumatol 1988: 15: 233-7.
5 Smith M D, Ahern M J, Brooks P M, et al. The clinical and immunological effects of pulse methylprednisolone therapy in ? rheumatoid arthritis. III. Effects on immune and inflammatory indices in synovial fluid. $J$ Rheumatol 1988; 15: 238-41.

6 Walters M T, Cawley M I D. Combined suppressive drug 0 treatment in severe refractory rheumatoid disease: an analysis $\bar{O}$ of the relative effects of parenteral methylprednisolone, cyclo- $\bar{N}$ phosphamide, and sodium aurothiomalate. Ann Rheum Dis 1988; 47: 924-9.

7 Williams I A, Mitchell A D, Rothman W, Tallett P, Williams of $K$, Pitt $P$. Survey of the long term incidence of osteonecrosis of $S$ the hip and adverse medical events in rheumatoid arthritis $\vec{\circ}$ after high dose intravenous methylprednisolone. Ann Rheum. Dis 1988; 47: 930-3.

8 Sebaldt R J. Pulse steroid therapy and the search for improved drug therapy of RA. J Rheumatol 1988; 15: 200-1.

9 Kraus A. Pulse steroid therapy, rheumatoid arthritis and the Journal of Rheumatology. J Rheumatol 1988; 15: 1872-3.

10 Sebaldt R J. Reply. J Rheumatol 1988; 15: 1873.

11 Smith M D, Ahern M J, Roberts-Thomson P J. Pulse steroid 6 therapy in rheumatoid arthritis: Can equivalent doses of oral $\infty$ prednisolone give similar clinical results to intravenous methyl- $\bullet$ prednisolone? Ann Rheum Dis 1988; 47: 28-33.

\section{Evaluating drugs in rheumatoid arthritis}

SIR, In their recent viewpoint Scott et al discussed the problems of assessing rheumatoid arthritis in clinicap trials. ${ }^{1}$ Some of these prominent workers have appareptly changed their minds lately. Some standard variables were not discussed.

The authors did not comment on the use of vis̄ial analogue scales, McConkey's clinical score, Ritchte' index, and patient opinion. Patient opinion is certainly one of the best methods of assessing the value of diseas $\overline{5}$ modifying drugs in rheumatoid arthritis. As long as thछे patient is taking a medicine he or she has accepted it, and the length of the treatment period is a measure of patient opinion of the drug. The time has passed when the doctor' opinion was the deciding factor for the patient. The reasons for withdrawal are often multifactorial, and factor other than those reported may be concealed. If the drug is. good for the patient he or she will stick to it and accept a) certain degree of inconvenience. Thus in my opinio treatment survival as a measure of patient opinion is the most important variable in an evaluation of any ant? rheumatic drug.

The authors stated that 'the debate about the true place of $x$ rays using current technology has subsided; there is now a relatively negative view of $x$ rays and less value is placed on them'. This view was not held in recent trials of methotrexate ${ }^{2}$ and auranofin, ${ }^{3}$ where $x$ ray evaluation waș of basic importance. Recently, Symmons and Dawes stated that radiological assessment is useful both in serial studies to assess the severity of disease and as an outcoms measure. ${ }^{4}$ Previously, McConkey had stated that in hiș opinion the best way of studying drugs in rheumatoig arthritis is to carry out serial measurements of the acute. phase proteins, relating these measurements to changes function and to changes in the radiographic appearance.

Rheumatology Department, ARVI LARSEN Västra sjukhuset,

72123 Västerås, Sweden

(n)

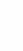

